Review

\title{
Blood-based Markers in the Prognostic Prediction of Esophagogastric Junction Cancer
}

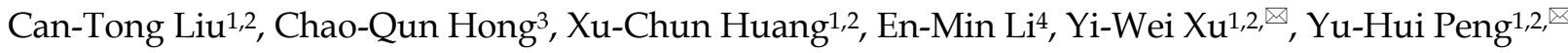 \\ 1. Department of Clinical Laboratory Medicine, the Cancer Hospital of Shantou University Medical College, Shantou, Guangdong, China \\ 2. Precision Medicine Research Center, Shantou University Medical College, Shantou, Guangdong, China \\ 3. Department of Oncological Laboratory Research, the Cancer Hospital of Shantou University Medical College, Shantou, Guangdong, China \\ 4. Department of Biochemistry and Molecular Biology, Shantou University Medical College, Shantou, Guangdong, China
}

$\square$ Corresponding authors: Yu-Hui Peng, Department of Clinical Laboratory Medicine, the Cancer Hospital of Shantou University Medical College, 7 Raoping Road, Shantou 515041, Guangdong, China. E-mail: pengyuhui666@163.com; Telephone: +86-137-1591-2739; Fax: +86-754-8856-0352. Also correspondence to Yi-Wei Xu, yiwei512@126.com

(c) The author(s). This is an open access article distributed under the terms of the Creative Commons Attribution License (https://creativecommons.org/licenses/by/4.0/). See http://ivyspring.com/terms for full terms and conditions.

Received: 2020.02.04; Accepted: 2020.04.13; Published: 2020.04.27

\begin{abstract}
Esophagogastric junction cancer poses a great threat to human beings both in western countries and East Asia, especially in China and Japan, and its incidence has increased during recent decades. The 5-year survival rate of esophagogastric junction cancer is quite poor compared with that of other gastric cancer sites. Until now, the traditional TNM staging system has been widely used in clinical practice for prognosis. However, the TNM system is based on pathology after surgical resection or radiology using CT and MRI, not on blood markers. Evidently, some research has been reported concentrated on the prognostic value of blood-based markers with the character of non-invasive and non-radioactive in EJA. Hematologic, biochemical and coagulation parameters could be obtained from clinical data and utilized to analyze their prognostic values. Tumor-associated antigens, microRNAs and circulating tumor cells have also been reported in EJC prognosis. In this article, we review research focused on blood-based markers to evaluate their prognostic value in esophagogastric junction cancer, especially its main subtype adenocarcinoma.
\end{abstract}

Key words: esophagogastric junction cancer, blood-based marker, prognosis

\section{Introduction}

Esophageal cancer and gastric cancer are two common malignant diseases, ranking sixth and ninth, respectively, in the incidence of cancers worldwide [1]. Esophagogastric junction cancer (EJC), whose main type is adenocarcinoma (EJA), is a malignant tumor with the center located within a 10-centimeter distance between the esophagus and stomach. In recent decades, the incidences of these two cancers have decreased, but EJC has instead increased in both East Asia and western countries [2]. EJC was first described by Siewert in 1998 [3] and has unique biological characteristics. Gastroesophageal reflux disease and Helicobacter pylori are associated with the increased risk of suffering from EJC [4, 5], and Barrett's esophagus (BE) is recognized as the precancerous lesion of adenocarcinoma in western countries [6].
The clinical manifestation of most patients suffering from EJC is dysphagia, which only becomes symptomatic at an advanced stage. With asymptomatic characteristics and the unpopularity of endoscope screening for early-stage EJC, Chinese patients tend to be diagnosed in the advanced stage [7]. Serosal invasion, lymph node metastasis and hematogenous recurrence are more likely to appear in EJC compared with the distant gastric cancer [8], which might be the reason why the 5-year overall survival (OS) rates of advanced stage EJC patients, who had undergone curative therapy, is less than $30 \%[9]$, lower than that of cancers occurring in other sites of the stomach. Although chemoradiotherapy does assist in improving the survival time in locally advanced EJC, the 5-year OS rates still remain low, ranging from $23 \%$ to $38 \%$ [10]. 
The American Joint Committee on Cancer Eighth Edition Cancer Staging Manual is widely used to predict the probable survival rate of esophageal cancer and EJC [11]. When staging EJC, tumors with centers no more than 2 centimeters into the gastric cardia are staged as esophageal carcinomas, while those with more than 2 centimeters are staged as gastric cancers. The latter used to be named gastric cardia cancer, the Siewert type III. The traditional TNM staging system, containing invasive depth, regional node metastasis and distant metastasis, is based on pathology after surgery or endoscopy, or computerized tomography and magnetic resonance imaging. When determining whether distant metastasis occurs, positron emission tomography is usually used. However, not included is any information from blood, an easily accessed, non-invasive and non-radioactive source.

Blood can be used to evaluate inflammation and nutritional status by testing its contents. After centrifugation, evaluation in serum and plasma of tissue function, such as liver and renal function, and coagulation function, can be determined. In the case of tumors, tumor-associated RNAs, proteins or cells, recognized as tumor-associated markers, will be released into the peripheral blood and can be utilized to assist in diagnosis and determination of the prognosis of cancers [12]. Positive detection of tumor candidates might indicate the existence of cancers, and their different concentrations might lead to different survival times. Recent concerns have arisen in the area of prognostic analysis of EJC based on blood-based markers. Here, we review relevant literatures on the value of blood-based markers for prognostic prediction in EJC.

\section{Hematologic Parameters}

The complete blood cell count (CBC) is a common method for evaluating inflammation and nutritional status. It can be completed in a few minutes after sampling without a complex and expensive facility. Therefore, its use is widespread in community hospitals. In the last few years, inflammation has been accepted as a hallmark in cancer progression and prognosis, and it can be evaluated with blood parameters, such as leukocytes [13]. Some parameters, including neutrophils and lymphocytes, have been discovered to be prognostic factors in many cancers [14]. Erythrocytes and platelets are generated from marrow, and their related parameters can show the function of marrow hematopoiesis, hinting at potential prognostic value of tumors.

From Figure 1A, among research involving CBC, the neutrophil-lymphocyte ratio (NLR) and platelet-lymphocyte ratio (PLR) are two of the most popular criteria in predicting prognosis of EJC. As shown in Table 1, increased NLR is one of the most frequently observed markers in EJC [15-18]. With cutoff values varying from $1.84-4.00$, the NLR might act as a potential marker in predicting the survival rate of patients with EJC [19-26], especially for patients who have undergone surgery. The NLR has been found to be correlated to tumor size [21], age [22] and $\mathrm{T}$ stage [24]. Although most of these studies involved a small sample size of patients, Wang et al. conducted a large-sample study (435 EJA patients in 1498 gastroesophageal adenocarcinoma patients) and showed that pretreatment NLR, as a continuous variable, can predict cancer-specific survival (CSS) independently in resectable EJA patients regardless of whether or not patients received neoadjuvant therapy [24]. Moreover, Zhang et al. found that a NLR value higher than 3.5 independently led to a poor overall survival of Siewert type II/III EJA (355 EJA patients) [21]. A larger study (611 EJA patients) performed by Zhang et al. suggested that NLR was associated with CSS, but does not play a vital role in predicting CSS of Siewert type II/III EJA[27]. Among these studies, NLR was correlated with $\mathrm{T}$ and $\mathrm{N}$ stages [24] and patients with NLR higher than 3 had a short overall survival time in stages IIB and III [22]. Therefore, it is important to further explore the predictive value of the NLR for predicting prognosis of EJA in a large-sample and multi-center study.

Thrombosis is frequent in cancer patients, resulting in high morbidity and mortality [28], and platelets participate in the process. Platelets coordinate in the immune system and affect cancer-related inflammation by changing the activation status of the endothelium and recruiting leukocytes to tumor sites [29]. It is reported that lymphocytes are vital for cancer immune-surveillance and immune-editing [30]. PLR, a combination of platelets and lymphocytes, has been found to be a prognostic factor in different cancers [31, 32]. In EJA patients receiving neoadjuvant therapy, Messager et al. found that an elevated PLR (PLR > 192) is associated independently with decreased disease-free survival (DFS; hazard ratio $[\mathrm{HR}]=2.85,95 \% \mathrm{CI}: 1.54$ 5.26, $p=0.001$ ) and overall survival (OS; HR $=2.47$, 95\% CI: $1.21-5.01, p=0.012$ ) [33]. Another study suggested a significant $p$-value of PLR $(p=0.038)$ in univariate analysis, but failed to further evaluate the independent probability [34]. Nevertheless, Zhou et al. conducted a retrospective study on EJA patients who underwent radical surgery to find that it was the higher preoperative lymphocyte-monocyte ratio (LMR), not NLR or PLR, that independently predicts poor OS [35]. 


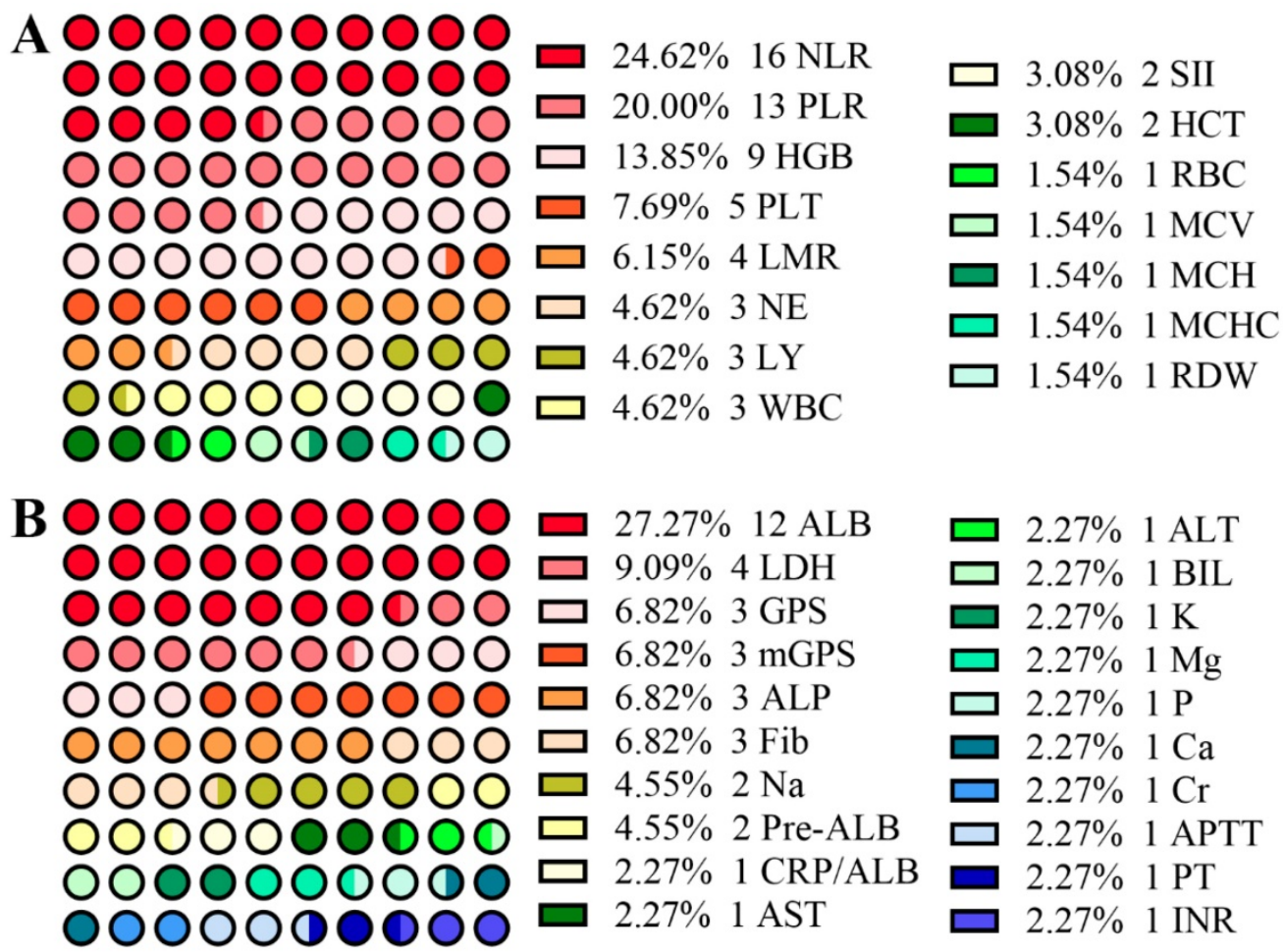

Figure 1. Dot diagrams of the number of studies involving blood-based hematologic parameters (A), and biochemical and coagulation parameters (B).

There were a few studies focusing on the association between absolute neutrophil (NE), lymphocyte (LY) or platelet (PLT) counts and EJC prognosis [26, 34, 36-38]. However, only Fuchs et al. found that abnormally low blood levels of LY (HR = 1.31, 95\% CI: $1.05-1.63, p=0.0015)$ and high levels of $\mathrm{NE}(\mathrm{HR}=1.52,95 \% \mathrm{CI}: 1.17-1.99, p<0.0001)$ were both candidates for predicting risk of EJC patients who underwent 4-month, first-line chemotherapy (platinum and/or fluoropyrimidine with or without an anthracycline) [37]. When combining these three parameters, a systemic immune-inflammation score (SII) has emerged, calculated by using a formula (SII = NE $\times$ LY/PLT), first described in 2014 to explore its prognostic value in hepatocellular carcinoma [39]. Jomrich et al. also introduced it for EJA and found that a higher SII contributes to poor OS and DFS in EJA patients who underwent esophagectomy with or without receiving neoadjuvant treatment [40].

With the occurrence of gastrointestinal bleeding, injury or aplastic anemia, RBCs will decrease, as well as hemoglobin (HGB). In a multicenter randomized trial including 248 EJC patients, an HGB lower than $110 \mathrm{~g} / 1$ has been excluded from the baseline prognostic model, although it showed significantly poor quality of life [38]. However, another study from China, conducted by Zhu et al., found that an HGB over $130 \mathrm{~g} / 1$ might be a protective marker for EJA, but not in other gastric cancers [41], which was not in accordance with a previous study involving only stage I and II patients [42]. Thus, a hierarchical analysis in different stages is provably needed. When turning to HGB- or RBC-related factors, few studies have been reported for EJC. Jomrich et al. evaluated the prognostic value of preoperative mean corpuscular volume (MCV), mean corpuscular hemoglobin $(\mathrm{MCH})$, mean corpuscular hemoglobin concentration (MCHC), and red blood cell distribution width (RDW) for patients with resectable EJC. For all patients, elevated $\mathrm{MCV}, \mathrm{MCH}$, and MCHC remained highly associated with reduced OS and DFS, and Cox regression analysis showed they could be independent prognostic factors in all EJC patients, but only MCV made sense in both OS and DFS in patients who were given neoadjuvant treatment [43]. In consideration of the delicate relationship between $\mathrm{MCH}$ and alcohol consumption in ESCC [44], the potential mechanism between MCV and alcohol in EJC might be another focus in the future.

\section{Biochemical and Coagulation Parameters}

Biochemical detection is popular in clinical practice. For example, high levels of alanine aminotransferase (ALT), aspartate aminotransferase (AST) and hypoalbuminemia usually indicate impairment of liver function. Hypoalbuminemia might result from reduced consumption. As mentioned before, the characteristic symptom of EJC is dysphagia, which will lead to a smaller diet and 
ultimately decreased albumin (ALB). The more serious the dysphagia, the lower the serum ALB. Thus, ALB might be a potential predictive marker for EJC. In fact, from Table 2, setting $35 \mathrm{~g} / 1$ as the cutoff value, four studies all showed that the low preoperative albumin, the most popular research subject (Figure 1B), can be a potentially independent marker for predicting poor survival of EJC[37, 45-47].
As an acute-phase protein with shorter half-life (about 1.9 days) than ALB, pre-albumin, a $54 \mathrm{kDa}$ protein, has become another focus of research. Han et al. and Zhang et al., from one research team, showed that a high level of pre-albumin could predict longer OS in EJA patients with Siewert type II and III who received gastrectomy [21, 48].

Table 1. Blood-based hematologic parameters in EJC prognosis

\begin{tabular}{|c|c|c|c|c|c|c|c|}
\hline Variables & Authors & Number of EJC Patients & Cutoff Values & Survival Types & Hazard Ratio & 95\% Confidence Interval & P-Value \\
\hline \multirow[t]{22}{*}{ NLR } & Cong X[19] & 129 & 1.84 & OS & 1.820 & $1.316-2.517$ & $\mathrm{a}<0.001$ \\
\hline & \multirow[t]{2}{*}{ Conway AM[22] } & \multirow[t]{2}{*}{316} & \multirow[t]{2}{*}{3} & TTP & 1.48 & $1.09-2.03$ & 0.013 \\
\hline & & & & OS & 1.56 & $1.15-2.11$ & 0.005 \\
\hline & \multirow[t]{2}{*}{ Custodio A[20] } & \multirow[t]{2}{*}{155} & 4 & OS & 1.2086 & $1.0366-1.4091$ & 0.0155 \\
\hline & & & 8 & OS & 1.4598 & $1.1177-1.9064$ & 0.0055 \\
\hline & Grenader T[25] & 227 & 3 & Os & 1.67 & $1.45-1.93$ & $<0.001$ \\
\hline & Jagadesham VP[34] & 105 & 2.78 & MS & - & - & $\mathrm{d} 0.061$ \\
\hline & \multirow[t]{2}{*}{ Jomrich G[40] } & \multirow[t]{2}{*}{320} & \multirow[t]{2}{*}{2.07} & OS & - & - & $<0.05$ \\
\hline & & & & DFS & - & - & $<0.05$ \\
\hline & Kudou K[49] & 59 & 2.26 & OS & 3.069 & $1.420-7.157$ & $\mathrm{a} 0.0041$ \\
\hline & \multirow[t]{2}{*}{ Noble F[26] } & \multirow[t]{2}{*}{138} & \multirow[t]{2}{*}{2.5} & OS & 1.191 & $1.092-1.298$ & $<0.0001$ \\
\hline & & & & DFS & 1.070 & $0.958-1.194$ & 0.230 \\
\hline & Tianxing G[56] & 129 & 1.89 & OS & 0.985 & $0.669-1.388$ & 0.930 \\
\hline & \multirow[t]{2}{*}{ Urabe M[59] } & \multirow[t]{2}{*}{87} & \multirow[t]{2}{*}{ con } & OS & 0.97 & $0.89-1.07$ & 0.56 \\
\hline & & & & DFS & 1.01 & $0.92-1.10$ & 0.87 \\
\hline & Wang SC[24] & 435 & con & CSS & 1.10 & $1.05-1.13$ & $<0.0001$ \\
\hline & Wang $\mathrm{Y}[60]$ & 215 & 2.2 & OS & 1.118 & $0.805-1.550$ & b0.506 \\
\hline & Yuan D[23] & 327 & 5 & OS & 2.551 & $1.847-3.524$ & $<0.0001$ \\
\hline & & & & DFS & 2.743 & $2.073-3.630$ & $<0.0001$ \\
\hline & Zhang JW[27] & 611 & 2.22 & CSS & 1.00 & $0.94-1.07$ & $b_{-}$ \\
\hline & Zhang L[21] & 355 & 3.5 & OS & 2.303 & $1.617-3.280$ & 0.000 \\
\hline & Zhou WJ[35] & 309 & 1.697 & OS & - & - & $b>0.05$ \\
\hline PLR & Cong X[19] & 129 & 110 & OS & 1.238 & $0.807-1.900$ & 0.327 \\
\hline & Jagadesham VP[34] & 105 & 158 & MS & - & - & $\mathrm{d} 0.038$ \\
\hline & Jomrich G[40] & 320 & 146.8 & OS & - & - & $<0.05$ \\
\hline & & & & DFS & - & - & $<0.05$ \\
\hline & Kudou K[49] & 59 & 165 & OS & 1.971 & $0.909-4.160$ & 0.0843 \\
\hline & Messager M[33] & 56 & 192 & OS & 2.47 & $1.21-5.01$ & 0.012 \\
\hline & & & & DFS & 2.85 & $1.54-5.26$ & 0.001 \\
\hline & Noble F[26] & 138 & 132.36 & OS & 1.002 & $1.000-1.005$ & 0.056 \\
\hline & & & & DFS & 1.000 & $0.997-1.003$ & 0.841 \\
\hline & Tianxing G[56] & 129 & - & OS & 1.396 & $0.843-2.311$ & 0.194 \\
\hline & Urabe M[59] & 87 & con & OS & 1.01 & $0.86-1.19$ & 0.90 \\
\hline & & & & DFS & 0.97 & $0.84-1.13$ & 0.73 \\
\hline & Wang Y[60] & 215 & 130.8 & OS & 1.256 & $0.905-1.742$ & b0.173 \\
\hline & Yuan D[23] & 327 & 150 & OS & 1.284 & $0.897-1.838$ & b0.172 \\
\hline & & & 300 & OS & 1.398 & $0.872-2.241$ & b0.164 \\
\hline & & & 150 & DFS & 1.338 & $0.979-1.829$ & b0.068 \\
\hline & & & 300 & DFS & 1.352 & $0.887-2.062$ & b0.161 \\
\hline & Zhang JW[27] & 611 & 124.4 & CSS & 1.00 & $1.00-1.00$ & - \\
\hline & Zhang L[21] & 355 & 171 & OS & 1.124 & $0.789-1.062$ & 0.517 \\
\hline & Zhou WJ[35] & 309 & 96.960 & OS & 1.188 & $0.795-1.775$ & 0.402 \\
\hline LMR & Cong X[19] & 129 & 3.25 & Os & 0.820 & $0.576-1.167$ & 0.271 \\
\hline & Urabe M[59] & 87 & con & Os & 0.98 & $0.91-1.06$ & 0.64 \\
\hline & & & & DFS & 0.98 & $0.92-1.06$ & 0.68 \\
\hline & Zhang JW[27] & 611 & 0.223 & CSS & 2.68 & $0.85-8.43$ & 0.092 \\
\hline & Zhou WJ[35] & 309 & 0.201 & OS & 1.604 & $1.071-2.402$ & 0.022 \\
\hline SII & Cong X[19] & 129 & 451 & OS & 1.040 & $0.668-1.618$ & 0.863 \\
\hline & Jomrich G[40] & 320 & 644 & OS & - & - & $<0.001$ \\
\hline & & & & DFS & - & - & $<0.001$ \\
\hline PLT & Bando H[36] & 14 & 150 & OS & - & - & 0.76 \\
\hline & Chau I[38] & 248 & median & OS & 0.955 & $0.839-1.086$ & b0.482 \\
\hline & Jagadesham VP[34] & 105 & 275 & MS & - & - & $\mathrm{b} 0.425$ \\
\hline & Noble F[26] & 138 & 226 & OS & 1.000 & $0.997-1.003$ & 0.837 \\
\hline & & & & DFS & 1.000 & $0.997-1.002$ & 0.761 \\
\hline & Yuan D[23] & 327 & - & OS & 1.045 & $0.835-1.308$ & b0.701 \\
\hline & & & & DFS & 1.033 & $0.846-1.260$ & b0.752 \\
\hline $\mathrm{NE}$ & Fuchs CS[37] & - & - & OS & 1.52 & $1.17-1.99$ & $<0.0001$ \\
\hline & Noble F[26] & 138 & 4 & OS & - & - & 0.811 \\
\hline
\end{tabular}




\begin{tabular}{|c|c|c|c|c|c|c|c|}
\hline Variables & Authors & Number of EJC Patients & Cutoff Values & Survival Types & Hazard Ratio & 95\% Confidence Interval & P-Value \\
\hline & & & & DFS & 1.096 & $0.972-1.237$ & 0.136 \\
\hline & Yuan D[23] & 327 & - & OS & 1.110 & $0.901-1.368$ & $\mathrm{~b} 0.328$ \\
\hline & & & & DFS & 1.184 & $0.985-1.424$ & $\mathrm{~b} 0.073$ \\
\hline \multirow[t]{5}{*}{ LY } & Fuchs CS[37] & - & - & OS & c1.31 & $1.05-1.63$ & 0.0015 \\
\hline & Noble F[26] & 138 & 1.7 & OS & 0.885 & $0.687-1.139$ & 0.342 \\
\hline & & & & DFS & 1.036 & $0.845-1.271$ & 0.731 \\
\hline & Yuan D[23] & 327 & - & OS & 0.838 & $0.648-1.083$ & b0.177 \\
\hline & & & & DFS & 0.810 & $0.650-1.011$ & b0.062 \\
\hline \multirow[t]{5}{*}{ WBC } & Chau I[38] & 248 & - & Os & - & - & 0.06 \\
\hline & Noble F[26] & 138 & - & Os & 1.074 & $0.982-1.175$ & 0.118 \\
\hline & & & & DFS & 1.063 & $0.968-1.167$ & 0.200 \\
\hline & Yuan D[23] & 327 & - & OS & 0.977 & $0.764-1.246$ & b0.850 \\
\hline & & & & DFS & 1.027 & $0.829-1.272$ & b0 0.807 \\
\hline \multirow[t]{9}{*}{ HGB } & Bando $\mathrm{H}[36]$ & 14 & $100 \mathrm{~g} / 1$ & OS & - & - & b0.127 \\
\hline & Chau I[38] & 248 & $110 \mathrm{~g} / 1$ & OS & - & - & 0.011 \\
\hline & Han WX[48] & 101 & $120 \mathrm{~g} / 1$ & Os & 1.000 & $0.527-1.899$ & 1.000 \\
\hline & Jomrich G[43] & 314 & - & OS & 0.98 & $0.90-1.06$ & 0.591 \\
\hline & & & & DFS & 0.99 & $0.92-1.07$ & 0.775 \\
\hline & Larsen AC[47] & 170 & - & OS & - & - & $b_{-}$ \\
\hline & Tianxing G[56] & 129 & - & OS & - & - & b0.095 \\
\hline & Zhang L[21] & 355 & $120 \mathrm{~g} / 1$ & OS & 0.943 & $0.671-1.318$ & 0.730 \\
\hline & Zhu Z[41] & 239 & $130 \mathrm{~g} / 1$ & Os & 0.689 & $0.501-0.946$ & 0.021 \\
\hline \multirow[t]{2}{*}{$\mathrm{MCV}$} & Jomrich G[43] & 314 & - & Os & 1.05 & $1.03-1.08$ & $<0.001$ \\
\hline & & & & DFS & 1.05 & $1.03-1.08$ & $<0.001$ \\
\hline \multirow[t]{2}{*}{$\mathrm{MCH}$} & Jomrich G[43] & 314 & - & OS & 1.14 & $1.07-1.22$ & $<0.001$ \\
\hline & & & & DFS & 1.12 & $1.05-1.20$ & $<0.001$ \\
\hline \multirow[t]{2}{*}{$\mathrm{MCHC}$} & Jomrich G[43] & 314 & - & OS & 1.17 & $1.07-1.28$ & 0.001 \\
\hline & & & & DFS & 1.17 & $1.07-1.27$ & $<0.001$ \\
\hline \multirow[t]{2}{*}{ RDW } & Jomrich G[43] & 314 & - & OS & 0.98 & $0.93-1.04$ & 0.538 \\
\hline & & & & DFS & 0.99 & 0.94-1.05 & 0.794 \\
\hline HCT & Cao HL[45] & 156 & - & OS & c5.353 & $3.419-8.380$ & $<0.001$ \\
\hline
\end{tabular}

EJC: esophagogastric junction cancer; NLR: neutrophil-lymphocyte ratio; PLR: platelet-lymphocyte ratio; LMR: lymphocyte-monocyte ratio; SII: systemic immune-inflammation score; PLT: platelet; NE: neutrophil count; LY: lymphocyte count; WBC: white blood cell; HGB: hemoglobin; MCV: mean corpuscular volume; MCH: mean corpuscular hemoglobin; MCHC: mean corpuscular hemoglobin concentration; RDW: red blood cell distribution width; HCT: hematocrit; OS: overall survival; DFS: disease-free survival; CSS: cancer-specific survival; TTP: time to progression; MS: median survival; con: continuous variable

a statistical significance in univariate analysis; ${ }^{\mathrm{b}}$ no statistical significance in univariate analysis; ${ }^{\mathrm{c}}$ the HR of low level; ${ }^{\mathrm{d}}$ not included in the multivariate analysis Note: the units for PLT, NE, LY and WBC are $10^{9} / 1$; the unit for RBC is $10^{12} / 1$.

A team from the Royal Marsden Hospital (RMH) conducted three randomized, controlled trials, and built a prognostic model using performance status, liver metastases, peritoneal metastasis, and alkaline phosphatase (ALP), to assess survival time in patients with locally advanced or metastatic EJA patients who underwent different chemotherapies [38]. In this RMH prognostic system, an ALP over $100 \mathrm{U} / 1$ hinted at poor survival time and quality of life. It also correlated with a significantly reduced probability of tumor response to chemotherapy. Another study from the Yale Cancer Center recruited more than 1,000 patients with gastric cancer or EJC and collected 41 baseline factors, including biochemical and coagulation parameters [37]. They found that high ALP, lactate dehydrogenase (LDH) and AST levels, and low albumin and sodium levels were independent markers for predicting poor OS. Meanwhile, another prognostic model was built based on 7 blood-based markers and other factors besides peritoneal metastases and Eastern Cooperative Oncology Group performance scores. The patients listed in these two models were ones with advanced cancers who received chemotherapy. However, there are fewer models based on blood markers in early-stage patients or patients with resectable EJA.

C-reactive protein (CRP) is an acute protein that rises sharply in plasma when the body is infected or damaged due to any type of inflammation. After activating complement, it can strengthen phagocytosis by phagocytes to play a complementary role, and clears away pathogenic microorganisms that invade the body and tissue cells that are damaged, necrotic and apoptotic. Combining CRP and ALB, CRP/ALB and Glasgow Prognostic Score (GPS) has been reported to assess EJC survival. Kudou et al. found that it was the CRP/ALB, but not GPS, that was strongly associated with poor OS in patients who underwent surgery for EJC [49]. Patients with high T stages or $\mathrm{N}$ stages preferred to contain a larger CRP/ALB which indicated poor RFS and OS. Compared with GPS, patients with a normal CRP level $(\leq 1.0 \mathrm{mg} / \mathrm{dl})$ regardless of albumin were given a modified GPS (mGPS) of 0[50]. Jomrich et al. thought that post-neoadjuvant therapy mGPS is highly associated with OS and DFS in patients suffering from neoadjuvantly-treated EJA (HR $=1.72,95 \%$ CI: 1.10 2.67 for OS; HR: 1.65, 95\% CI: 1.08 - 2.50 for DFS) [46]. A research from China also determined its prognostic value in predicting OS and DFS in EJA patients with resection [51]. Park et al. suggested that mGPS might 
be an independent marker for survival in patients with EJA (163 out of 203 participants, including gastric cancer) undergoing palliative self-expandable metallic stent insertion (HR $=1.24,95 \%$ CI: 1.03 - 1.49) [52].

\section{Combination of CBC and Biochemical or Coagulation Parameters}

The controlling nutritional status (CONUT) score is calculated from the serum albumin, total cholesterol, and absolute lymphocyte count [53], and better predicts survival than NLR and GPS in gastric cancer. However, it might not be a significant independent prognostic marker in EJA patients after surgery [49]. Due to the small amount of research, a further study concentrating on CONUT scores to evaluate the prognostic value of EJA is needed.

Fibrinogen is a protein involved in clotting and thrombosis, and synthesized by the liver [54]. Hyperfibrinogenemia has been seen to correlate with cancer progression and poor survival in colon cancer [55]. In limited EJC research, there has been little concern about fibrinogen alone. A novel scoring system, denoted F-NLR, has recently aroused some attention. Patients with both hyperfibrinogenemia ( $\geq$ $3.09 \mathrm{~g} / \mathrm{l})$ and high NLR $(\geq 1.89)$ were given a score of 2 , while ones with neither hyperfibrinogenemia nor high NLR were given a score of 0 .

Table 2. Blood-based biochemical and coagulation parameters in EJC prognosis

\begin{tabular}{|c|c|c|c|c|c|c|c|}
\hline Variables & Authors & Number of EJC patients & Cutoff Values & Survival Types & Hazard Ratio & 95\% Confidence Interval & $P$-Value \\
\hline \multirow[t]{14}{*}{ ALB } & Bando H[36] & 14 & $35 \mathrm{~g} / 1$ & OS & - & - & $<0.001$ \\
\hline & Cao HL[45] & 156 & $35 \mathrm{~g} / 1$ & Os & c1.907 & $1.058-3.438$ & 0.032 \\
\hline & Chau I[38] & 248 & median & OS & 0.686 & $0.597-0.790$ & $\mathrm{a}<0.0001$ \\
\hline & Custodio A[20] & 155 & LLN & - & - & - & - \\
\hline & Fuchs CS[37] & - & - & OS & c1.33 & $1.07-1.65$ & 0.0006 \\
\hline & Han WX[48] & 101 & $40 \mathrm{~g} / 1$ & Os & 0.945 & $0.469-1.903$ & 0.874 \\
\hline & Jomrich G[46] & 155 & $35 \mathrm{~g} / 1$ & OS & 0.52 & $0.33-0.82$ & 0.005 \\
\hline & & & & DFS & 0.51 & $0.33-0.80$ & 0.004 \\
\hline & Larsen AC[47] & 170 & - & OS & - & - & $b_{-}$ \\
\hline & Noble F[26] & 138 & $35 \mathrm{~g} / 1$ & OS & - & - & 0.137 \\
\hline & & & & DFS & 0.957 & $0.919-0.997$ & 0.034 \\
\hline & Tianxing G[56] & - & $42 \mathrm{~g} / 1$ & Os & - & - & b0.725 \\
\hline & Zhang L[21] & 355 & $40 \mathrm{~g} / 1$ & Os & - & - & 0.061 \\
\hline & Zhu Z[41] & 239 & $40 \mathrm{~g} / 1$ & OS & - & - & b0.946 \\
\hline \multirow[t]{2}{*}{ Pre-ALB } & Han WX[48] & 101 & $200 \mathrm{~g} / 1$ & OS & 0.494 & $0.271-0.901$ & 0.021 \\
\hline & Zhang L[21] & 355 & $180 \mathrm{~g} / 1$ & OS & 0.428 & $0.310-0.592$ & 0.000 \\
\hline BIL & Custodio A[20] & 155 & ULN & - & - & - & - \\
\hline \multirow[t]{3}{*}{ ALP } & Chau I[38] & 248 & $100 \mathrm{U} / 1$ & OS & 1.412 & $1.136-1.755$ & $<0.0001$ \\
\hline & Custodio A[20] & 155 & ULN & - & - & - & - \\
\hline & Fuchs CS[37] & - & & OS & 1.28 & $1.03-1.60$ & 0.0030 \\
\hline \multirow[t]{4}{*}{ LDH } & Bando H[36] & 14 & ULN & OS & - & - & $\mathrm{a}<0.001$ \\
\hline & Custodio A[20] & 155 & ULN & - & - & - & - \\
\hline & Fuchs CS[37] & - & & OS & 1.31 & $1.05-1.63$ & 0.0019 \\
\hline & Larsen AC[47] & 170 & - & OS & 3.03 & $1.54-5.94$ & 0.001 \\
\hline AST & Fuchs CS[37] & - & - & Os & 1.37 & $1.06-1.76$ & 0.0014 \\
\hline \multirow[t]{2}{*}{$\mathrm{Na}$} & Chau I[38] & 248 & median & OS & 0.721 & $0.621-0.837$ & $a<0.0001$ \\
\hline & Fuchs CS[37] & - & - & OS & c2.04 & $1.54-2.71$ & $<0.0001$ \\
\hline $\mathrm{Ca}$ & Chau I[38] & 248 & median & OS & 1.005 & $0.856-1.178$ & b0.956 \\
\hline \multirow[t]{6}{*}{ GPS } & Cui Y[51] & 332 & 1 & OS & 2.32 & $1.69-3.20$ & $<0.001$ \\
\hline & & & & DFS & 2.36 & $1.73-3.22$ & $<0.001$ \\
\hline & & & 2 & OS & 5.08 & $3.01-8.57$ & $<0.001$ \\
\hline & & & & DFS & 3.01 & $1.71-5.29$ & $<0.001$ \\
\hline & Kudou K[49] & 59 & 1 & OS & 3.758 & $1.556-8.234$ & $\mathrm{a} 0.0047$ \\
\hline & Jagadesham VP[34] & 105 & 1 & MS & 1.58 & $0.62-4.06$ & 0.337 \\
\hline \multirow[t]{7}{*}{ mGPS } & Jomrich G[46] & 155 & $1+2$ & OS & 1.72 & $1.10-2.67$ & 0.017 \\
\hline & & & $1+2$ & DFS & 1.65 & $1.08-2.50$ & 0.0195 \\
\hline & Park JH[52] & 163 & $1 / 2$ & OS & 1.24 & $1.03-1.49$ & 0.021 \\
\hline & Urabe M[59] & 87 & 1 & Os & 1.08 & $0.64-1.70$ & b0.093 \\
\hline & & & 2 & OS & 2.11 & $1.08-3.69$ & b0.093 \\
\hline & & & 1 & DFS & 1.07 & $0.65-1.66$ & 0.081 \\
\hline & & & 2 & DFS & 0.49 & $0.25-0.89$ & 0.081 \\
\hline CRP/ALB & Kudou K[49] & 59 & 0.1 & OS & 2.378 & $1.025-5.249$ & 0.0439 \\
\hline \multirow[t]{3}{*}{$\mathrm{Fib}$} & Cong $X[19]$ & 129 & $3.09 \mathrm{~g} / 1$ & Os & 2.598 & $1.851-3.645$ & $a<0.001$ \\
\hline & Jagadesham VP[34] & 105 & $4.9 \mu \mathrm{mol} / \mathrm{l}$ & MS & - & - & $\mathrm{d} 0.005$ \\
\hline & Tianxing G[56] & 129 & $3.09 \mathrm{~g} / 1$ & Os & 1.083 & $0.696-1.684$ & 0.724 \\
\hline
\end{tabular}

EJC: esophagogastric junction cancer; ALB: albumin; Pre-ALB: pre-albumin; BIL: bilirubin; ALP: alkaline phosphatase; LDH: lactate dehydrogenase; AST: aspartate aminotransferase; Na: sodium; Ca: calcium; GPS: Glasgow prognostic score; mGPS: modified GPS; Fib: fibrinogen; LLN: lower limit of normal; ULN: upper limit of normal; OS: overall survival; DFS: disease-free survival; MS: median survival

${ }^{a}$ statistical significance in univariate analysis; b no statistical significance in univariate analysis; ${ }^{c}$ the HR of low level; ${ }^{d}$ not included in the multivariate analysis 
Table 3. Combination of hematologic, biochemical and coagulate parameters in EJC prognosis

\begin{tabular}{|c|c|c|c|c|c|c|c|}
\hline Variables & Authors & Number of EJC Patients & Cutoff Values & Survival Types & Hazard Ratio & 95\% Confidence Interval & $P$-Value \\
\hline CONUT score & Kudou K[49] & 59 & 3 & OS & 4.749 & $2.146-10.09$ & $\mathrm{a} 0.0003$ \\
\hline \multirow[t]{3}{*}{ F-NLR } & Cong X[19] & 129 & 1 & OS & 1.921 & $1.124-3.283$ & 0.017 \\
\hline & & & 2 & OS & 2.764 & $1.559-4.900$ & 0.001 \\
\hline & Tianxing G[56] & 129 & - & OS & 1.730 & $1.173-2.551$ & 0.006 \\
\hline \multirow[t]{6}{*}{ PNI } & Han WX[48] & 101 & 51 & OS & 0.751 & $0.372-1.518$ & 0.426 \\
\hline & Noble F[26] & 138 & 47.50 & OS & - & - & 0.323 \\
\hline & & & & DFS & 0.979 & $0.950-1.009$ & 0.165 \\
\hline & Urabe M[59] & 112 & con & OS & 0.62 & $0.47-0.82$ & $<0.001$ \\
\hline & & & & DFS & 0.60 & $0.46-0.78$ & $<0.001$ \\
\hline & Zhang L[21] & 355 & 51.3 & OS & 1.192 & $0.828-1.715$ & 0.345 \\
\hline AGR/PNI & Wang Y[60] & 215 & $1 / 2$ & OS & 0.613 & $0.226-0.923$ & $<0.001$ \\
\hline
\end{tabular}

EJC: esophagogastric junction cancer; CONUT score: controlling nutritional status score; F-NLR: combination of fibrinogen concentration and neutrophil-lymphocyte ratio; PNI: prognostic nutritional index; AGR: albumin-to-globulin ratio; OS: overall survival; DFS: disease-free survival; con: continuous variable

a statistical significance in univariate analysis

As shown in Table 3, both studies acquired the same results in which F-NLR could be an independent factor for predicting OS of EJA patients $[19,56]$. Cong et al. conducted a training-validation cohort study and found the area under the receiver operating characteristic curve of F-NLR in predicting the survival of EJC was 0.717 (95\% CI: 0.664 - 0.770), slightly higher than that of TNM staging $(0.700 ; 95 \%$ CI: $0.646-0.754)$, although there was no statistical difference[19]. When stratified by pathological TNM staging, the OS of EJA patients with F-NLR 2 was poor compared with that of F-NLR 0 or 1 both in stages I - II and in stages III (all $p<0.001$ in the combined set). In addition, Tianxing et al. found that F-NLR was associated with tumor size and TNM stage (both $p<0.01$ ) [56].

First described by Pennsylvania researchers [57] and revised by Japanese researchers [58], the prognostic nutritional index (PNI) is another parameter containing $\mathrm{CBC}$ and biochemical indices. It can be calculated from the serum albumin concentration $(\mathrm{g} / \mathrm{l})$ plus five times the absolute lymphocyte counts $\left(\times 10^{9} / 1\right)$. It can mirror malnutrition status due to the impaired digestive function, such as dysphagia and loss of appetite. Four studies included PNI (Table 3) [21, 26, 48, 59], but only Urabe et al. was able to show that preoperative PNI is independently associated with OS and relapse-free survival (HR $=0.62,95 \% \mathrm{CI}: 0.47-0.82, p<0.001$; HR $=0.60,95 \%$ CI: $0.46-0.78, p<0.001$, respectively) [59] in 1363 patients who underwent surgery with gastric cancer with a small sample size of 87 EJA patients. When stratifying PNI into four groups in which patients with PNI larger than 51.9 in the fourth quartile, the authors found that constituent ratios of PNI differed in different $\mathrm{T}$ stages and $\mathrm{N}$ stages. Thus, a definite relationship between PNI and EJA survival still remains to be shown. Wang et al. tried to combine the albumin-to-globulin ratio (AGR) and PNI to establish an innovative system to estimate its prognostic value in Siewert type III EJA, and found that AGR-PNI is associated with age, tumor size, NLR and PLR (all $p<0.05$ ), serving as an independent predictor for OS of EJA patients[60]. Although there was no statistically significant relationship between AGR-PNI and pathological TNM stage $(p=0.607)$, patients with AGR-PNI 1 or 2 had better OS rates in stages I+II and III than that with AGR-PNI 3.

\section{Tumor-Associated Circulating Materials}

External and internal antigens stimulate our immune system to secrete antibodies [61]. Cancer can express and release tumor-associated antigens into the circulating environment, so detection of their serum levels should assist in estimating the occurrence of malignancy, response to therapy and prognosis. Carcinoembryonic antigen (CEA) and carbohydrate antigen 19-9 (CA19-9) have been utilized for several decades as major serum tumor markers for gastrointestinal cancers. It is reported that elevated preoperative serum CEA and CA19-9 correlate with poor survival in pancreatic cancer [62]. Tokunaga et al. tried to evaluate the prognostic value of CEA and CA19-9 in EJA [63]. As a result, both them were found to be associated with depth of invasion and lymph node metastasis (all $p<0.05$ ) and a high level of both could imply an advanced stage. However, in univariate and multivariate analysis, only CA19-9 served as a useful prognostic factor in patients with EJA (for CSS: HR $=3.89,95 \%$ CI: 1.41 10.33; for OS: HR = 2.43, 95\% CI: 1.03 - 5.35). Recently, a review highly commented the value of autoantibodies in the detection of esophageal cancer and EJA [64], but there lacks related studies using autoantibodies to discuss their accuracy in predicting survival time of EJA patients.

Tumorigenesis and metastasis usually partner with angiogenesis, which relies on both angiogenic and growth factors $[65,66]$. Using enzyme-linked immunosorbent assay, Park et al. initially detected the serum levels of several preoperative angiogenic factors, including vascular endothelial growth factor A (VEGF-A), fibroblast growth factor 2 (FGF2), epidermal growth factor (EGF) and hepatocyte 
growth factor (HGF), in patients with gastric cancer and EJA who underwent gastrectomy or esophagogastrectomy [67], and built an adjusted total value (ATV) uniting four factors. When these four factors were taken into consideration, multivariate analysis showed that only VEGF-A was a statistically significant independent prognostic factor for OS $(p=$ 0.028 ) while ATV remained a powerful factor $(p=$ 0.013 ) in another model taking into account margin status, tumor size, T category, N category and ATV. Bevacizumab is a monoclonal antibody that can inhibit VEGF, and is used for treating various metastatic cancers, including metastatic colorectal cancer and non-small-cell lung cancer. Thus, the potential of bevacizumab united with platinum in advanced EJA might be a good combination to improve survival.

Messenger RNA (mRNA) is transcribed from DNA and is translated into protein, evoking an opinion that they appear earlier than the tumor-associated proteins. Using quantitative real-time polymerase chain reaction, Qiao et al. suggested that enhanced cytokeratin 19 and CEA mRNA levels are related to lymph node metastasis. Increased pre-cytokeratin 19 and CEA mRNA levels were independent prognostic factors for OS in gastric cardia cancer patients receiving surgery [68]. As noncoding 17- to 25-nucleotide-long RNA, microRNA has been seen as a new type of marker for numerous diseases, and plays vital roles in tumorigenesis, metastasis and prognosis [69]. $\mathrm{Yu}$ et al. investigated the expression of microRNA and identified a five-microRNA signature, including hsa-let-7a, hsa-miR-221, hsa-miR-137, hsa-miR-372, and hsa-miR-182, as a novel independent prognostic factor in non-small-cell lung cancer patients [70]. In the EJA field, Odenthal et al. showed, in 50 patients with local advanced EJA who underwent neoadjuvant therapy followed by surgical resection, that 122 microRNAs were differentially expressed between healthy volunteers and EJA participants [71]. They indicated that high miR-302c and low miR-222 expression were significantly correlated with better OS. These two studies based on blood-based RNA verify the feasibility of using tumor markers in blood for predicting survival of EJA patients.

Circulating DNA or RNA methylation test is a research hotspot in the recent year in different cancers, such as colorectal cancer [72], hepatocellular carcinoma [73], breast cancer [74] and so on. When it came to EJC, Guo et al. detected the aberrant methylation status of long coding RNA LOC100130476 in peripheral white blood cells in three regions, different parts in exon or intron [75]. Patients with region 1 (located in exon 1: from +245 to $+413 \mathrm{bp}$ ) hypermethylation of LOC100130476 revealed significant poorer 5-year survival rates compared with those with region 1 unmethylation of the marker $(\mathrm{P}<0.05)$. The Cox multivariate analysis showed that the methylation of region 1 might be an independent prognostic marker of gastric cardia adenocarcinoma.

Circulating tumor cells (CTCs), which can be derived from the primary tumor and enter into the circulation with the potential for metastasis, are another target of intense research in cancer, especially in advanced cancers. Among patients with metastatic EJA, Kubisch et al. isolated CTCs from peripheral blood of 62 patients (25 patients with EJA) and detected their mRNA levels [76]. Results showed that the presence of CTCs was a predictor for OS and progression-free survival, and the mRNA transcripts were associated with tumor survival.

\section{Conclusion and Perspectives}

Prognosis of esophagogastric junction cancer is poor. Only the traditional TNM staging system is utilized to evaluate the prognosis and treatment decision. Novel markers are urgently needed for assistant. Among we reviewed here, NLR, a popular object of study, is widely seen as a potential prognostic predictive marker. When combined with fibrinogen, F-NLR, functioned as another prognostic marker, was verified by two research teams $[19,56]$. The limitation of small account and single center indicated the requirement of more study. When it turns to biochemical indices, albumin and LDH might act as meaningful markers in predicting survival time of EJC.

Epstein-Barr (EB) virus, a gamma-herpesvirus, is found to be related to several diseases, such as infectious mononucleosis [77], Burkitt's lymphoma [78] and nasopharyngeal carcinoma [65]. EB virus also infect the gastric epithelial cell, might leading to gastric carcinoma, which takes a nine percent in all gastric cancers [79]. Although Wang et al. thought that EB virus could be associated with esophageal squamous cell carcinoma[80], most of other research hold the same view of no pertinence in esophageal carcinogenesis [81, 82]. Genitsch et al. found a low positive detection of EB virus-encoded small RNAs in tumor samples of EJC patients [83]. However, the detection in peripheral blood of EJC patients is absent. Thus, it is urgently needed to explore the association between circulating EB virus antigen, antibodies or RNA and EJC.

The purpose of this review is to illuminate recent work on the predictive value of blood-based markers for prognosis in EJC. If cancer-related RNAs, proteins and cells can be taken into consideration, the accuracy for determining EJC prognosis can be enhanced. The 
methylation of gene might be a novel and hotspot. Moreover, related research should be completed besides the concise mechanism which is needed for elucidating how they work on the development of EJC. Most of the enrolled studies focused on the pretreatment blood markers, but not in the post-treatment fields. With the characteristics of low-cost and minimally invasive techniques, after additional verification, blood-based markers might brighten the future of treatment options for EJC.

\section{Abbreviations}

EJC: esophagogastric junction cancer; EJA: esophagogastric junction adenocarcinoma; $\mathrm{BE}$ : Barrett's esophagus; NLR: neutrophil-lymphocyte ratio; PLR: platelet-lymphocyte ratio; LMR: lymphocyte-monocyte ratio; SII: systemic immune-inflammation score; PLT: platelet; NE: neutrophil count; LY: lymphocyte count; WBC: white blood cell; HGB: hemoglobin; $\mathrm{MCV}$ : mean corpuscular volume; $\mathrm{MCH}$ : mean corpuscular hemoglobin; MCHC: mean corpuscular hemoglobin concentration; RDW: red blood cell distribution width; HCT: hematocrit; OS: overall survival; DFS: disease-free survival; CSS: cancer-specific survival; TTP: time to progression; MS: median survival; ALB: albumin; Pre-ALB: pre-albumin; BIL: bilirubin; ALP: alkaline phosphatase; LDH: lactate dehydrogenase; AST: aspartate aminotransferase; Na: sodium; Ca: calcium; GPS: Glasgow prognostic score; mGPS: modified GPS; Fib: fibrinogen; LLN: lower limit of normal; ULN: upper limit of normal; CONUT score: controlling nutritional status score; F-NLR: combination of fibrinogen concentration and neutrophil-lymphocyte ratio; PNI: prognostic nutritional index; AGR: albumin-to-globulin ratio; EB virus: Epstein-Barr virus.

\section{Acknowledgements}

This work was supported by grants from the Natural Science Foundation of China (grant numbers 81972801), the Natural Science Foundation of Guangdong Province (grant numbers 2018A0303 07079, 2019A1515011873), the Innovative and Strong School Project of Guangdong (grant number 2018KTSCX068); and Grant for Key Disciplinary Project of Clinical Medicine under the Guangdong High-level University Development Program. And we thank Stanley $\mathrm{Li}$ for the proofread of this manuscript.

\section{Competing Interests}

The authors have declared that no competing interest exists.

\section{References}

1. Bray F, Ferlay J, Soerjomataram I, Siegel RL, Torre LA, Jemal A. Global cancer statistics 2018: GLOBOCAN estimates of incidence and mortality worldwide for 36 cancers in 185 countries. CA Cancer J Clin. 2018; 68: 394-424.

2. Arnold M, Soerjomataram I, Ferlay J, Forman D. Global incidence of oesophageal cancer by histological subtype in 2012. Gut. 2015; 64: 381-7.

3. Siewert JR, Stein HJ. Classification of adenocarcinoma of the oesophagogastric junction. Br J Surg. 1998; 85: 1457-9.

4. Enzinger PC, Mayer RJ. Esophageal cancer. N Engl J Med. 2003; 349: 2241-52.

5. McColl KE, Going JJ. Aetiology and classification of adenocarcinoma of the gastro-oesophageal junction/cardia. Gut. 2010; 59: 282-4.

6. Sharma P. Clinical practice. Barrett's esophagus. N Engl J Med. 2009; 361: 2548-56.

7. Chen W, Zheng R, Baade PD, Zhang S, Zeng H, Bray F, et al. Cancer statistics in China, 2015. CA Cancer J Clin. 2016; 66: 115-32.

8. Saito H, Fukumoto Y, Osaki T, Fukuda K, Tatebe S, Tsujitani S, et al. Distinct recurrence pattern and outcome of adenocarcinoma of the gastric cardia in comparison with carcinoma of other regions of the stomach. World J Surg. 2006; 30: 1864-9.

9. Haga $Y$, Hato S, Ikenaga M, Yamamoto K, Tsuburaya A, Doi K, et al. Validation of an assessment tool: Estimation of Postoperative Overall Survival for Gastric Cancer. Eur J Surg Oncol. 2018; 44: 515-23.

10. Fontana E, Smyth EC, Cunningham D. Esophagogastric Adenocarcinoma: Is More Chemotherapy Better? Curr Treat Options Oncol. 2016; 17: 21.

11. Rice TW, Gress DM, Patil DT, Hofstetter WL, Kelsen DP, Blackstone EH. Cancer of the esophagus and esophagogastric junction-Major changes in the American Joint Committee on Cancer eighth edition cancer staging manual. CA Cancer J Clin. 2017; 67: 304-17.

12. Shaw A, Bradley MD, Elyan S, Kurian KM. Tumour biomarkers: diagnostic, prognostic, and predictive. BMJ. 2015; 351: h3449.

13. Candido J, Hagemann T. Cancer-related inflammation. J Clin Immunol. 2013; 33 (Suppl 1): S79-84

14. Shaul ME, Fridlender ZG. Tumour-associated neutrophils in patients with cancer. Nat Rev Clin Oncol. 2019; 16: 601-20.

15. Lorente D, Mateo J, Templeton AJ, Zafeiriou Z, Bianchini D, Ferraldeschi R, et al. Baseline neutrophil-lymphocyte ratio (NLR) is associated with survival and response to treatment with second-line chemotherapy for advanced prostate cancer independent of baseline steroid use. Ann Oncol. 2015; 26: 750-5.

16. Lalani AA, Xie W, Martini DJ, Steinharter JA, Norton CK, Krajewski KM, et al. Change in Neutrophil-to-lymphocyte ratio (NLR) in response to immune checkpoint blockade for metastatic renal cell carcinoma. J Immunother Cancer. 2018; 6: 5 .

17. Bartlett EK, Flynn JR, Panageas KS, Ferraro RA, Sta Cruz JM, Postow MA, et al. High neutrophil-to-lymphocyte ratio (NLR) is associated with treatment failure and death in patients who have melanoma treated with PD-1 inhibitor monotherapy. Cancer. 2020; 126: 76-85.

18. Sunakawa Y, Yang D, Cao S, Zhang W, Moran $\mathrm{M}$, Astrow $\mathrm{SH}$, et al. Immune-related Genes to Dominate Neutrophil-lymphocyte Ratio (NLR) Associated With Survival of Cetuximab Treatment in Metastatic Colorectal Cancer. Clin Colorectal Cancer. 2018; 17: e741-e9.

19. Cong X, Li S, Zhang Y, Zhu Z, Wang Y, Song S, et al. The combination of preoperative fibrinogen and neutrophil-lymphocyte ratio is a predictive prognostic factor in esophagogastric junction and upper gastric cancer. J Cancer. 2019; 10: 5518-26.

20. Custodio A, Carmona-Bayonas A, Jimenez-Fonseca P, Sanchez ML, Viudez A, Hernandez R, et al. Nomogram-based prediction of survival in patients with advanced oesophagogastric adenocarcinoma receiving first-line chemotherapy: a multicenter prospective study in the era of trastuzumab. Br J Cancer. 2017; 116: 1526-35.

21. Zhang L, Su Y, Chen Z, Wei Z, Han W, Xu A. The prognostic value of preoperative inflammation-based prognostic scores and nutritional status for overall survival in resected patients with nonmetastatic Siewert type II/III adenocarcinoma of esophagogastric junction. Medicine (Baltimore). 2017; 96: e7647.

22. Conway AM, Salih Z, Papaxoinis G, Fletcher K, Weaver J, Patrao A, et al. Significance of blood neutrophil-to-lymphocyte ratio for prognostic stratification of patients with gastroesophageal junction adenocarcinoma in the era of the 8th edition of the American Joint Committee on Cancer (AJCC8) staging. Med Oncol. 2017; 34: 116.

23. Yuan $\mathrm{D}, \mathrm{Zhu} \mathrm{K}, \mathrm{Li}, \mathrm{K}$, Yan $\mathrm{R}$, Jia $\mathrm{Y}$, Dang $\mathrm{C}$ The preoperative neutrophil-lymphocyte ratio predicts recurrence and survival among patients undergoing R0 resections of adenocarcinomas of the esophagogastric junction. J Surg Oncol. 2014; 110: 333-40.

24. Wang SC, Chou JF, Strong VE, Brennan MF, Capanu M, Coit DG. Pretreatment Neutrophil to Lymphocyte Ratio Independently Predicts Disease-specific Survival in Resectable Gastroesophageal Junction and Gastric Adenocarcinoma. Ann Surg. 2016; 263: 292-7.

25. Grenader T, Waddell T, Peckitt C, Oates J, Starling N, Cunningham D, et al. Prognostic value of neutrophil-to-lymphocyte ratio in advanced oesophago-gastric cancer: exploratory analysis of the REAL-2 trial. Ann Oncol. 2016; 27: 687-92.

26. Noble F, Hopkins J, Curtis N, Kelly JJ, Bailey IS, Byrne JP, et al. The role of systemic inflammatory and nutritional blood-borne markers in predicting 
response to neoadjuvant chemotherapy and survival in oesophagogastric cancer. Med Oncol. 2013; 30: 596.

27. Zhang JW, Huang L, Xu AM. Preoperative monocyte-lymphocyte and neutrophil-lymphocyte but not platelet-lymphocyte ratios are predictive of clinical outcomes in resected patients with non-metastatic Siewert type II/III adenocarcinoma of esophagogastric junction: a prospective cohort study (the AMONP corhort). Oncotarget. 2017; 8: 57516-27.

28. Ruf W. Tissue factor and cancer. Thromb Res. 2012; 130 (Suppl 1): S84-7.

29. Olsson AK, Cedervall J. The pro-inflammatory role of platelets in cancer. Platelets. 2018; 29: 569-73.

30. Dunn GP, Old LJ, Schreiber RD. The immunobiology of cancer immunosurveillance and immunoediting. Immunity. 2004; 21: 137-48.

31. Cannon NA, Meyer J, Iyengar P, Ahn C, Westover KD, Choy H, et al. Neutrophil-lymphocyte and platelet-lymphocyte ratios as prognostic factors after stereotactic radiation therapy for early-stage non-small-cell lung cancer. J Thorac Oncol. 2015; 10: 280-5

32. Krenn-Pilko S, Langsenlehner U, Thurner EM, Stojakovic T, Pichler M, Gerger A, et al. The elevated preoperative platelet-to-lymphocyte ratio predicts poor prognosis in breast cancer patients. Br J Cancer. 2014; 110: 2524-30.

33. Messager M, Neofytou K, Chaudry MA, Allum WH. Prognostic impact of preoperative platelets to lymphocytes ratio (PLR) on survival for oesophageal and junctional carcinoma treated with neoadjuvant chemotherapy: A retrospective monocentric study on 153 patients. Eur J Surg Oncol. 2015; 41: $1316-23$

34. Jagadesham VP, Lagarde SM, Immanuel A, Griffin SM. Systemic inflammatory markers and outcome in patients with locally advanced adenocarcinoma of the oesophagus and gastro-oesophageal junction. Br J Surg. 2017; 104: 401-7.

35. Zhou WJ, Wu J, Li XD, Wang Q, Ni XF, Jiang JT, et al. [Effect of preoperative monocyte-lymphocyte ratio on prognosis of patients with resectable esophagogastric junction cancer]. Zhonghua Zhong Liu Za Zhi. 2017; 39: $178-83$.

36. Bando H, Rubinstein L, Harris P, Yoshino T, Doi T, Ohtsu A, et al. Analysis of esophagogastric cancer patients enrolled in the National Cancer Institute Cancer Therapy Evaluation Program sponsored phase 1 trials. Gastric Cancer. 2017; 20: 481-8.

37. Fuchs CS, Muro K, Tomasek J, Van Cutsem E, Cho JY, Oh SC, et al. Prognostic Factor Analysis of Overall Survival in Gastric Cancer from Two Phase III Studies of Second-line Ramucirumab (REGARD and RAINBOW) Using Pooled Patient Data. J Gastric Cancer. 2017; 17: 132-44.

38. Chau I, Norman AR, Cunningham D, Waters JS, Oates J, Ross PJ. Multivariate prognostic factor analysis in locally advanced and metastatic esophago-gastric cancer--pooled analysis from three multicenter, randomized, controlled trials using individual patient data. J Clin Oncol. 2004; 22: 2395-403.

39. Hu B, Yang XR, Xu Y, Sun YF, Sun C, Guo W, et al. Systemic immune-inflammation index predicts prognosis of patients after curative resection for hepatocellular carcinoma. Clin Cancer Res. 2014; 20: 6212-22.

40. Jomrich G, Paireder M, Kristo I, Baierl A, Ilhan-Mutlu A, Preusser M, et al. High Systemic Immune-Inflammation Index is an Adverse Prognostic Factor for Patients With Gastroesophageal Adenocarcinoma. Ann Surg 2019; [Epub ahead of print].

41. Zhu Z, Wang Y, Li F, Gao J, Han B, Wang R, et al. [Comparison of clinicopathological features and prognosis between adenocarcinoma of esophagogastric junction and adenocarcinoma of gastric antrum]. Zhonghua Wei Chang Wai Ke Za Zhi. 2019; 22: 149-55.

42. Shen JG, Cheong JH, Hyung WJ, Kim J, Choi SH, Noh SH. Pretreatment anemia is associated with poorer survival in patients with stage I and II gastric cancer. J Surg Oncol. 2005; 91: 126-30.

43. Jomrich G, Hollenstein M, John M, Ristl R, Paireder M, Kristo I, et al. High Mean Corpuscular Volume Predicts Poor Outcome for Patients With Gastroesophageal Adenocarcinoma. Ann Surg Oncol. 2019; 26: 976-85.

44. Zheng YZ, Dai SQ, Li W, Cao X, Li Y, Zhang LJ, et al. Prognostic value of preoperative mean corpuscular volume in esophageal squamous cell carcinoma. World J Gastroenterol. 2013; 19: 2811-7.

45. Cao HL, Jin SO, Wang KB, Bai B. Prognostic factors influencing the survival of patients with carcinoma of the gastric cardia receiving preoperative interventional embolization chemotherapy. Oncotarget. 2017; 8: 79567-75.

46. Jomrich G, Hollenstein M, John M, Baierl A, Paireder M, Kristo I, et al. The modified glasgow prognostic score is an independent prognostic indicator in neoadjuvantly treated adenocarcinoma of the esophagogastric junction. Oncotarget. 2018; 9: 6968-76.

47. Larsen AC, Hollander $\mathrm{C}$, Duval L, Schonnemann $\mathrm{K}$, Achiam M, Pfeiffer $\mathrm{P}$, et al. A nationwide retrospective study of perioperative chemotherapy for gastroesophageal adenocarcinoma: tolerability, outcome, and prognostic factors. Ann Surg Oncol. 2015; 22: 1540-7.

48. Han WX, Chen ZM, Wei ZJ, Xu AM. Preoperative pre-albumin predicts prognosis of patients after gastrectomy for adenocarcinoma of esophagogastric junction. World J Surg Oncol. 2016; 14: 279

49. Kudou K, Saeki H, Nakashima $Y$, Kamori T, Kawazoe T, Haruta $Y$, et al. C-reactive protein/albumin ratio is a poor prognostic factor of esophagogastric junction and upper gastric cancer. J Gastroenterol Hepatol. 2019; 34: 355-63.

50. McMillan DC. An inflammation-based prognostic score and its role in the nutrition-based management of patients with cancer. Proc Nutr Soc. 2008; 67: $257-62$.
51. Cui Y, Li J, Liu M, Shi Z, Fu Y, Cai L, et al. [Value of Glasgow prognostic score in patients with adenocarcinoma of esophagogastric junction]. Zhonghua Wei Chang Wai Ke Za Zhi. 2016; 19: 54-7.

52. Park JH, Woodley N, McMillan DC, Glen P. Palliative stenting for oesophagogastric cancer: tumour and host factors and prognosis. BMJ Support Palliat Care. 2019; 9: 332-9.

53. Kuroda D, Sawayama H, Kurashige J, Iwatsuki M, Eto T, Tokunaga R, et al. Controlling Nutritional Status (CONUT) score is a prognostic marker for gastric cancer patients after curative resection. Gastric Cancer. 2018; 21: 204-12.

54. de Moerloose P, Casini A, Neerman-Arbez M. Congenital fibrinogen disorders: an update. Semin Thromb Hemost. 2013; 39: 585-95.

55. Son HJ, Park JW, Chang HJ, Kim DY, Kim BC, Kim SY, et al. Preoperative plasma hyperfibrinogenemia is predictive of poor prognosis in patients with nonmetastatic colon cancer. Ann Surg Oncol. 2013; 20: 2908-13.

56. Tianxing G, Xiaojie P, Lihuan Z, Yangyun H. Combination of preoperative fibrinogen and neutrophil to lymphocyte ratio is a predictive prognostic factor in ESCC and AEG systematic review. Biosci Rep. 2019; 39; doi: 10.1042/BSR20190480.

57. Buzby GP, Mullen JL, Matthews DC, Hobbs CL, Rosato EF. Prognostic nutritional index in gastrointestinal surgery. Am J Surg. 1980; 139: 160-7.

58. Onodera T, Goseki N, Kosaki G. [Prognostic nutritional index in gastrointestinal surgery of malnourished cancer patients]. Nihon Geka Gakkai Zasshi. 1984; 85: 1001-5.

59. Urabe M, Yamashita H, Watanabe T, Seto Y. Comparison of Prognostic Abilities Among Preoperative Laboratory Data Indices in Patients with Resectable Gastric and Esophagogastric Junction Adenocarcinoma. World J Surg. 2018; 42: 185-94.

60. Wang Y, Zhu Z, Li C, Ma Y, You Q, Li Z, et al. Prognostic significance of preoperative albumin-to-globulin ratio and prognostic nutritional index combined score in Siewert type 3 adenocarcinoma of esophagogastric junction. Cancer Manag Res. 2019; 11: 7631-8

61. Wang L, Gao J, Lan X, Zhao H, Shang X, Tian F, et al. Identification of combined T-cell and B-cell reactive Echinococcus granulosus 95 antigens for the potential development of a multi-epitope vaccine. Ann Transl Med. 2019; 7: 652

62. Wu L, Huang P, Wang F, Li D, Xie E, Zhang Y, et al. Relationship between serum CA19-9 and CEA levels and prognosis of pancreatic cancer. Ann Transl Med. 2015; 3: 328.

63. Tokunaga R, Imamura $Y$, Nakamura K, Uchihara T, Ishimoto T, Nakagawa S, et al. Carbohydrate antigen 19-9 is a useful prognostic marker in esophagogastric junction adenocarcinoma. Cancer Med. 2015; 4: 1659-66.

64. Xu YW, Peng YH, Xu LY, Xie JJ, Li EM. Autoantibodies: Potential clinical applications in early detection of esophageal squamous cell carcinoma and esophagogastric junction adenocarcinoma. World J Gastroenterol. 2019; 25: 5049-68

65. Yang L, Liu L, Xu Z, Liao W, Feng D, Dong X, et al. EBV-LMP1 targeted DNAzyme enhances radiosensitivity by inhibiting tumor angiogenesis via the JNKs/HIF-1 pathway in nasopharyngeal carcinoma. Oncotarget. 2015; 6: 5804-17.

66. Shen L, Zhou Q, Wang Y, Liao W, Chen Y, Xu Z, et al. Antiangiogenic and antitumoral effects mediated by a vascular endothelial growth factor receptor 1 (VEGFR-1)-targeted DNAzyme. Mol Med. 2013; 19: 377-86.

67. Park DJ, Yoon C, Thomas N, Ku GY, Janjigian YY, Kelsen DP, et al. Prognostic significance of targetable angiogenic and growth factors in patients undergoing resection for gastric and gastroesophageal junction cancers. Ann Surg Oncol. 2014; 21: 1130-7.

68. Qiao YF, Chen CG, Yue J, Ma MQ, Ma Z, Yu ZT. Prognostic significance of preoperative and postoperative CK19 and CEA mRNA levels in peripheral blood of patients with gastric cardia cancer. World J Gastroenterol. 2017; 23: 1424-33.

69. Iorio MV, Croce CM. MicroRNAs in cancer: small molecules with a huge impact. J Clin Oncol. 2009; 27: 5848-56.

70. Yu SL, Chen HY, Chang GC, Chen CY, Chen HW, Singh S, et al. MicroRNA signature predicts survival and relapse in lung cancer. Cancer Cell. 2008; 13: 48-57.

71. Odenthal M, Hee J, Gockel I, Sisic L, Schmitz J, Stoecklein NH, et al. Serum microRNA profiles as prognostic/predictive markers in the multimodality therapy of locally advanced adenocarcinomas of the gastroesophageal unction. Int J Cancer. 2015; 137: 230-7.

72. Luo H, Zhao Q, Wei W, Zheng L, Yi S, Li G, et al. Circulating tumor DNA methylation profiles enable early diagnosis, prognosis prediction, and screening for colorectal cancer. Sci Transl Med. 2020; 12; doi: 10.1126 /scitranslmed.aax7533.

73. Xu RH, Wei W, Krawczyk M, Wang W, Luo H, Flagg K, et al. Circulating tumour DNA methylation markers for diagnosis and prognosis of hepatocellular carcinoma. Nat Mater. 2017; 16: 1155-61.

74. Visvanathan K, Fackler MS, Zhang Z, Lopez-Bujanda ZA, Jeter SC, Sokoll LJ, et al. Monitoring of Serum DNA Methylation as an Early Independent Marker of Response and Survival in Metastatic Breast Cancer: TBCRC 005 Prospective Biomarker Study. J Clin Oncol. 2017; 35: 751-8.

75. Guo W, Dong Z, Shi Y, Liu S, Liang J, Guo Y, et al. Methylation-mediated downregulation of long noncoding RNA LOC100130476 in gastric cardia adenocarcinoma. Clin Exp Metastasis. 2016: 33: 497-508.

76. Kubisch I, de Albuquerque A, Schuppan D, Kaul S, Schaich M, Stolzel U. Prognostic Role of a Multimarker Analysis of Circulating Tumor Cells in 
Advanced Gastric and Gastroesophageal Adenocarcinomas. Oncology. 2015; 89: 294-303.

77. Luzuriaga K, Sullivan JL. Infectious mononucleosis. N Engl J Med. 2010; 362: 1993-2000.

78. Young LS, Rickinson AB. Epstein-Barr virus: 40 years on. Nat Rev Cancer. 2004; 4: 757-68.

79. Farrell PJ. Epstein-Barr Virus and Cancer. Annu Rev Pathol. 2019; 14: 29-53.

80. Wang LS, Chow KC, Wu YC, Li WY, Huang MH. Detection of Epstein-Barr virus in esophageal squamous cell carcinoma in Taiwan. Am J Gastroenterol. 1999; 94: 2834-9.

81. Wang J, Noffsinger A, Stemmermann G, Fenoglio-Preiser C. Esophageal squamous cell carcinomas arising in patients from a high-risk area of North China lack an association with Epstein-Barr virus. Cancer Epidemiol Biomarkers Prev. 1999; 8: 1111-4.

82. Awerkiew S, zur Hausen A, Baldus SE, Holscher AH, Sidorenko SI, Kutsev SI, et al. Presence of Epstein-Barr virus in esophageal cancer is restricted to tumor infiltrating lymphocytes. Med Microbiol Immunol. 2005; 194: 187-91.

83. Genitsch V, Novotny A, Seiler CA, Kroll D, Walch A, Langer R. Epstein-barr virus in gastro-esophageal adenocarcinomas - single center experiences in the context of current literature. Front Oncol. 2015; 5: 73 